Supporting information for

\title{
Synthesis of a Chiral Crystal Form of MOF-5, CMOF-5, by Chiral Induction
}

\author{
Shi-Yuan Zhang, ${ }^{\dagger,}$ Dan Li, ${ }^{\S}$ Dong Guo,${ }^{\S}$ Hui Zhang, ${ }^{\S}$ Wei Shi, ${ }^{\ddagger}$ Peng Cheng, ${ }^{\ddagger}$ Lukasz \\ Wojtas, and Michael J. Zaworotko, $\dagger$ \\ ${ }^{\dagger}$ Department of Chemical \& Environmental Science, Materials and Surface Science Institute, University of \\ Limerick, Limerick, Republic of Ireland \\ tDepartment of Chemistry, Key Laboratory of Advanced Energy Materials Chemistry (MOE), \\ Collaborative Innovation Center of Chemical Science and Engineering, Nankai University, Tianjin 300071, \\ P. R. China \\ ${ }^{\S}$ Department of Chemistry, College of Chemistry and Chemical Engineering, Xiamen University, Xiamen \\ 361005, P. R. China \\ "Department of Chemistry, University of South Florida, 4202 East Fowler Avenue, CHE205, Tampa, \\ Florida 33620, United States
}




\section{Table of contents}

Section

Page(s)

Experimental section

S3-S5

Comparison of the structures of CMOF-5 and MOF-5 (Figure S1)

S5

Comparison of PXRD of CMOF-5 and MOF-5 (Figure S2)

S6

Comparison of IR spectra (Figure S3)

S7

TGA and $\mathrm{N}_{2}$ adsorption of CMOF-5 (Figures S4-S5)

S8

VCD spectra of $\Lambda$-CMOF-5 and $\Delta$-CMOF-5 (Figures S6-S7)

S9-S12

PXRD of solvent exchanged CMOF-5 (Figure S8)

S13

PXRD of phase transformation (Figure S9)

S14

VCD spectra of MOF-5 after NMP treatment (Figure S10)

S15-S16

Crystallographic table (Table S1)

S17 


\section{Experimental Section}

Materials and Measurements: All reagents and solvents were commercially available and used as received.

\section{X-ray diffraction}

Single-crystal X-ray diffraction data for $\Lambda$-CMOF-5 and $\Delta$-CMOF-5 were collected on a Bruker D8 Venture PHOTON $100 \mathrm{CMOS}$ system equipped with a $\mathrm{Cu} \mathrm{K} \alpha$ INCOATEC Imus micro-focus source $(\lambda=1.54178 \AA, T=100(2) \mathrm{K})$. The diffraction data for [Zn(BDC)(NMP)] were measured on a Bruker-AXS SMART APEX/CCD diffractometer using $\mathrm{Cu} \mathrm{K}_{\alpha}$ radiation $(\lambda=1.5418 \AA, T=$ 228(2) K). In all cases indexing was performed using APEX2. ${ }^{1}$ Data integration and reduction were performed using SaintPlus 6.01. ${ }^{2}$ Absorption correction was performed by multi-scan method implemented in SADABS. ${ }^{3}$ Space groups were determined using XPREP implemented in APEX2. ${ }^{1}$ Structures were solved using Patterson Method (SHELXS-97), expanded using Fourier methods and refined on $\mathrm{F}^{2}$ using nonlinear least-squares techniques with SHELXL-97 contained in APEX2 and WinGX v1.70.01 ${ }^{4-7}$ programs packages.

\section{$\Lambda$-CMOF-5, $\Delta$-CMOF-5 and [Zn(BDC)(NMP)]:}

The disorder of the ligand is observed in all three structures. Disordered atoms have generally been refined isotropically and for some cases with EADP constrains for very closely located atoms. Restraints have been used to refine the geometry of 1,4-BDC in all cases. All crystals were merohedrally twinned with 180 rotation about [011] as twinning law - (-1 00000010010$)$. In case of structure $\Delta$-CMOF-5, the reflection set has been detwinned using Shelxl 2013 and Squeeze procedure in PLATON ${ }^{9,10}$ has been used to account for disordered solvent. For structure $\Lambda$ CMOF-5, the unidentifiable solvent molecules were refined as oxygen atoms with fractional occupancy (Detwinning caused deterioration of the data and has not been performed). All structures could be solved in Pa-3 space group and refined to similar, as for $\mathrm{P} 2{ }_{1} 3, \mathrm{R}$-values. The Sohncke space group P2 33 has been chosen in accordance with VCD spectra suggesting that the crystals are chiral and other techniques suggesting, that there is no amino acid present in structural voids. The apparent centrosymmetricity could be caused by disorder. For [Zn(BDC)(NMP)], all non-hydrogen atoms were refined anisotropically. Hydrogen atoms were placed in geometrically calculated positions and included in the refinement process using riding model with isotropic thermal parameters. 
[1] Bruker (2010). (APEX2). Bruker AXS Inc., Madison, Wisconsin, USA.

[2] Bruker (2009). SAINT. Data Reduction Software. Bruker AXS Inc., Madison, Wisconsin, USA.

[3] Sheldrick, G. M. (2008). SADABS. Program for Empirical Absorption Correction. University of Gottingen, Germany.

[4] Farrugia L.J. Appl. Cryst. (1999). 32, 837-838

[5] Sheldrick, G.M. (1997) SHELXL-97. Program for the Refinement of Crystal.

[6] Sheldrick, G.M. (1990) Acta Cryst. A46, 467-473

[7] Sheldrick, G. M. (2008) Acta Cryst. A64, 112-122.

[8] Rohlicek J., Husak M. (2007) J. Appl. Cryst. 40, 600-601.

[9] Spek T.L. (1990) Acta Cryst. A46, 194-201.

[10] Spek T.L. (1990) Acta Cryst. A46, c34.

Powder X-Ray diffraction was performed on a Bruker D8 Advance $\theta / \theta$ diffractometer (Cu-K $\alpha, \lambda$ $=1.5418 \AA$ ) equipped with a 1D LynxEye Super Speed detector and $0.02 \mathrm{~mm}$ Ni filter. Elemental analyses (C, H and N) were performed on a Perkin-Elmer 240 CHN elemental analyzer. Thermogravimetric Analysis was performed using a TA Instruments TGA-Q50 at a constant rate of $5^{\circ} \mathrm{C} / \mathrm{min}$ from $25^{\circ} \mathrm{C}$ to $800^{\circ} \mathrm{C}$. IR spectra were recorded on a Perkin-Elmer Spectrum Two spectrometer. VCD spectra were measured using a ChiraIR Dual-PEM VCD spectrometer from BioTools. One single crystal was picked up and weighted (2 3mg), then mixed and grinded with dry $\mathrm{KBr}$ powder $(60 \sim 90 \mathrm{mg})$. Spectra were collected on powder samples of a crystal embedded in $\mathrm{KBr}$ pellets (1:30). Low pressure gas sorption isotherms were measured using a Micrometrics ASAP 2020 surface area and pore size analyzer. Prior to the measurements, the samples were exchanged with $\mathrm{CH}_{2} \mathrm{Cl}_{2}$ solvent 3 times per day for 3 days and were degassed for 1 day at $85{ }^{\circ} \mathrm{C}$. UHP grade nitrogen gas was used for measurement.

Synthesis of $\Lambda$-MOF-5 and $\Delta$-MOF-5. A mixture of $\mathrm{Zn}\left(\mathrm{NO}_{3}\right)_{2} \cdot 6 \mathrm{H}_{2} \mathrm{O}(60 \mathrm{mg}, 0.2 \mathrm{mmol})$, $\mathrm{H}_{2} \mathrm{BDC}$ (33 mg, $0.2 \mathrm{mmol}$ ), L-proline/D-proline (23 mg, $\left.0.2 \mathrm{mmol}\right)$, NMP (4 mL) and $\mathrm{H}_{2} \mathrm{O}(1 \mathrm{~mL})$ was sealed in a $20 \mathrm{~mL}$ glass vial and heated at $120{ }^{\circ} \mathrm{C}$ for 2 days, and then cooled to room temperature. Large cubic shape crystals for $\Lambda$-MOF-5 and $\Delta$-MOF-5 were obtained respectively, washed by NMP, and dried at room temperature. Yield: $84 \%$ based on $\mathrm{Zn}$. Chemical formula:

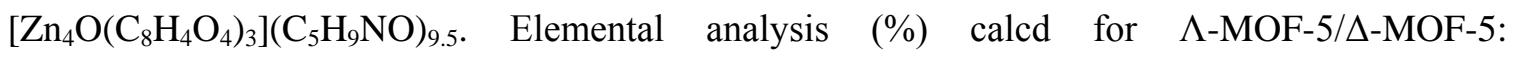
$\mathrm{C}_{71.5} \mathrm{H}_{97.5} \mathrm{~N}_{9.5} \mathrm{Zn}_{4} \mathrm{O}_{2}$ (1711.73), C, 50.17; H, 5.74; N, 7.77; found: C, 50.14; H, 5.39; N, 8.02. 
Synthesis of $[\mathbf{Z n}(\mathbf{B D C})(\mathbf{N M P})]$. The similar procedure was used as $\Lambda$-MOF-5 and $\Delta$-MOF-5 without L-proline or D-proline. Colorless tabular crystals were obtained. Chemical formula: $\left[\mathrm{Zn}\left(\mathrm{C}_{8} \mathrm{H}_{4} \mathrm{O}_{4}\right)\left(\mathrm{C}_{5} \mathrm{H}_{9} \mathrm{NO}\right)\right]$.

Synthesis of as-MOF-5. A mixture of $\mathrm{Zn}\left(\mathrm{NO}_{3}\right)_{2} \cdot 6 \mathrm{H}_{2} \mathrm{O}(60 \mathrm{mg}, 0.2 \mathrm{mmol}), \mathrm{H}_{2} \mathrm{BDC}(33 \mathrm{mg}, 0.2$ mmol) and DEF ( $5 \mathrm{~mL}$ ) was sealed in a $20 \mathrm{~mL}$ glass vial and heated at $135{ }^{\circ} \mathrm{C}$ for 2 days, and then cooled to room temperature. Large cubic shape crystals for as-MOF-5 were obtained.

Solvent exchange of CMOF-5. The crystals of $\Lambda$-MOF-5 were immersed in different solvent (2propanol, hexane, tetrahydrofuran, acetone, methanol, acetonitrile, dimethylformamide, dichloromethane, ethyl acetate, ethanol, and cyclohexane) for $24 \mathrm{~h}$. The transformations from CMOF-5 to MOF-5 were studied by power X-ray diffraction.

Phase transformation. The crystals of as-MOF-5 were immersed in dichloromethane for $4 \mathrm{~h}$. And then the crystals were filtered and immersed in NMP for 6h. Powder X-ray diffraction and VCD spectra were used to study the transformation of the crystals.

\section{Additional Figures and Table}

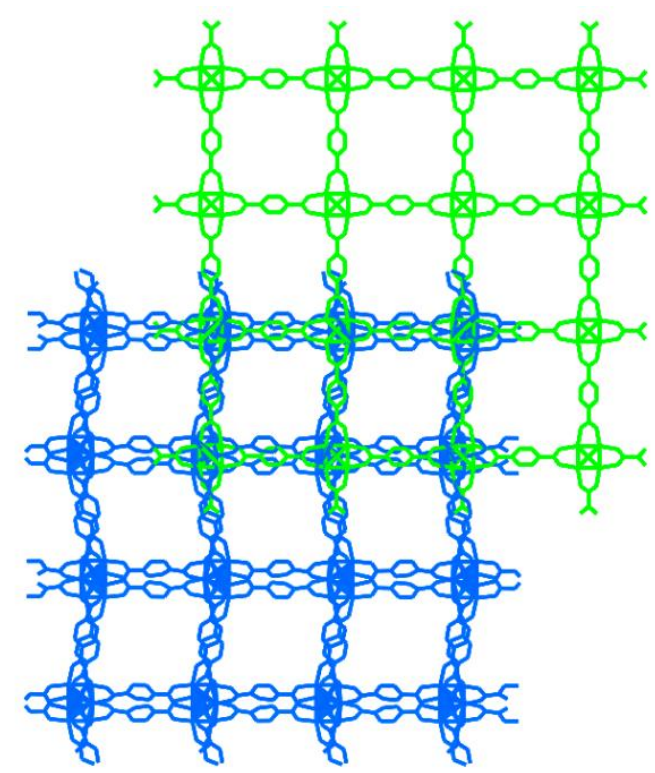

Figure S1. Comparison of the two crystal structures of CMOF-5 and MOF-5. The water wave like layer of CMOF-5 is result from the distorted of metal clusters and ligands. 


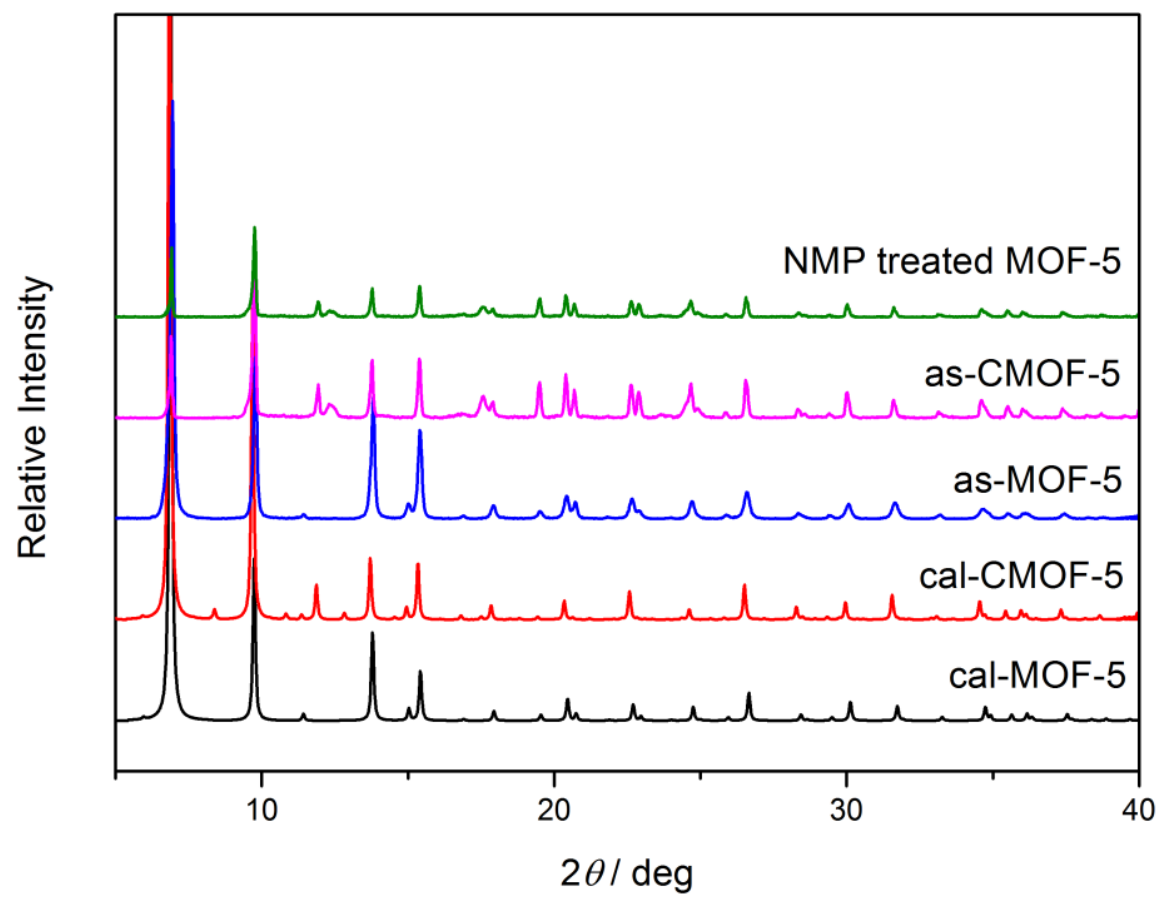

Figure S2. Comparison of experimental and calculated powder X-ray diffraction patterns of calMOF-5 (black), cal-CMOF-5 (red), as-MOF-5 (blue), as-CMOF-5 (magenta) and NMP treated MOF-5 (green). 


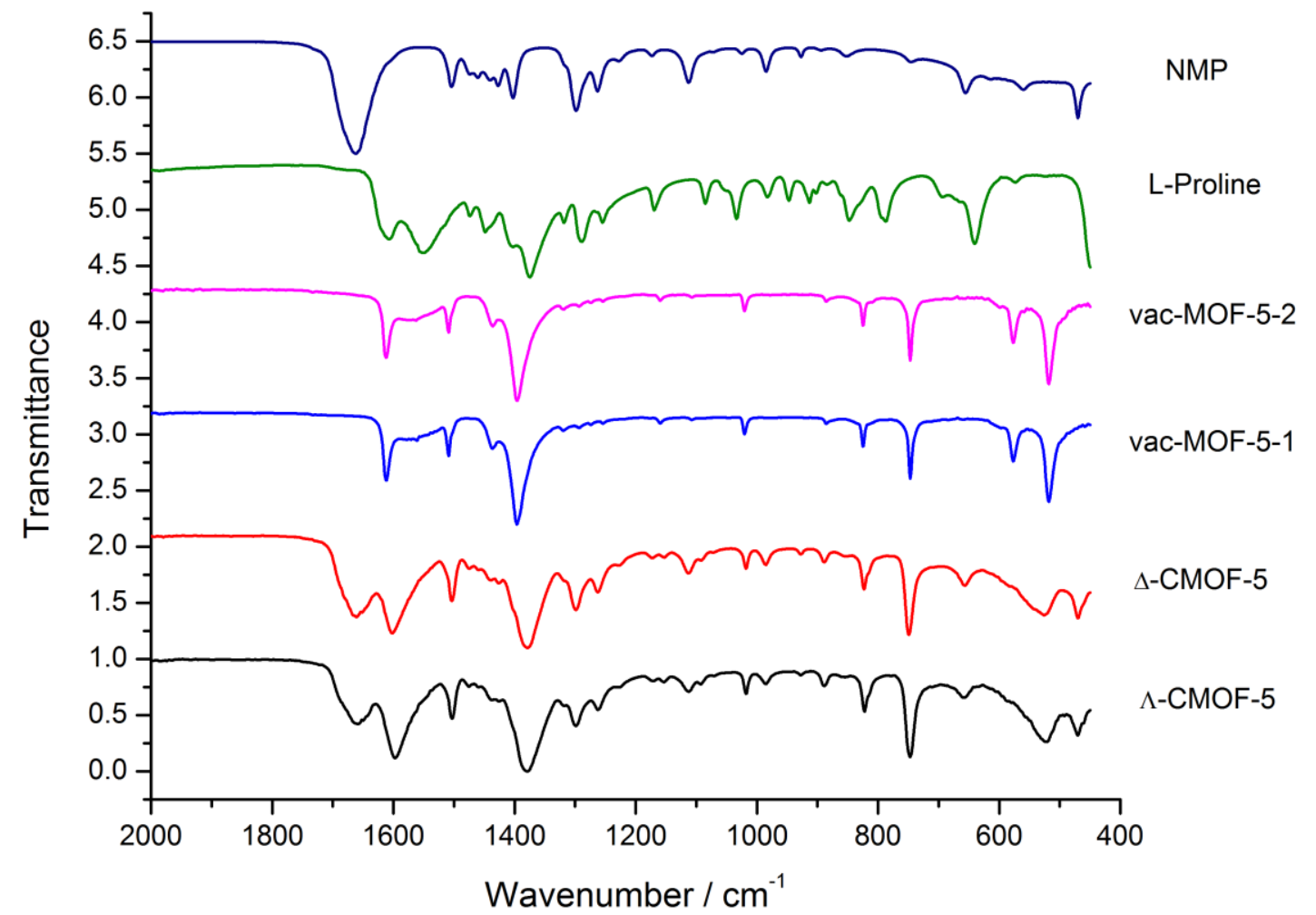

Figure S3. IR spectra of $\Lambda$-CMOF-5, $\Delta$-CMOF-5, vac-MOF-5-1, vac-MOF-5-2, L-proline and NMP. vac-MOF-5-1 and vac-MOF-5-2 were obtained after degassing of $\Lambda$-CMOF-5 and $\Delta$ CMOF-5, respectively, showing the same spectra of original MOF-5. 


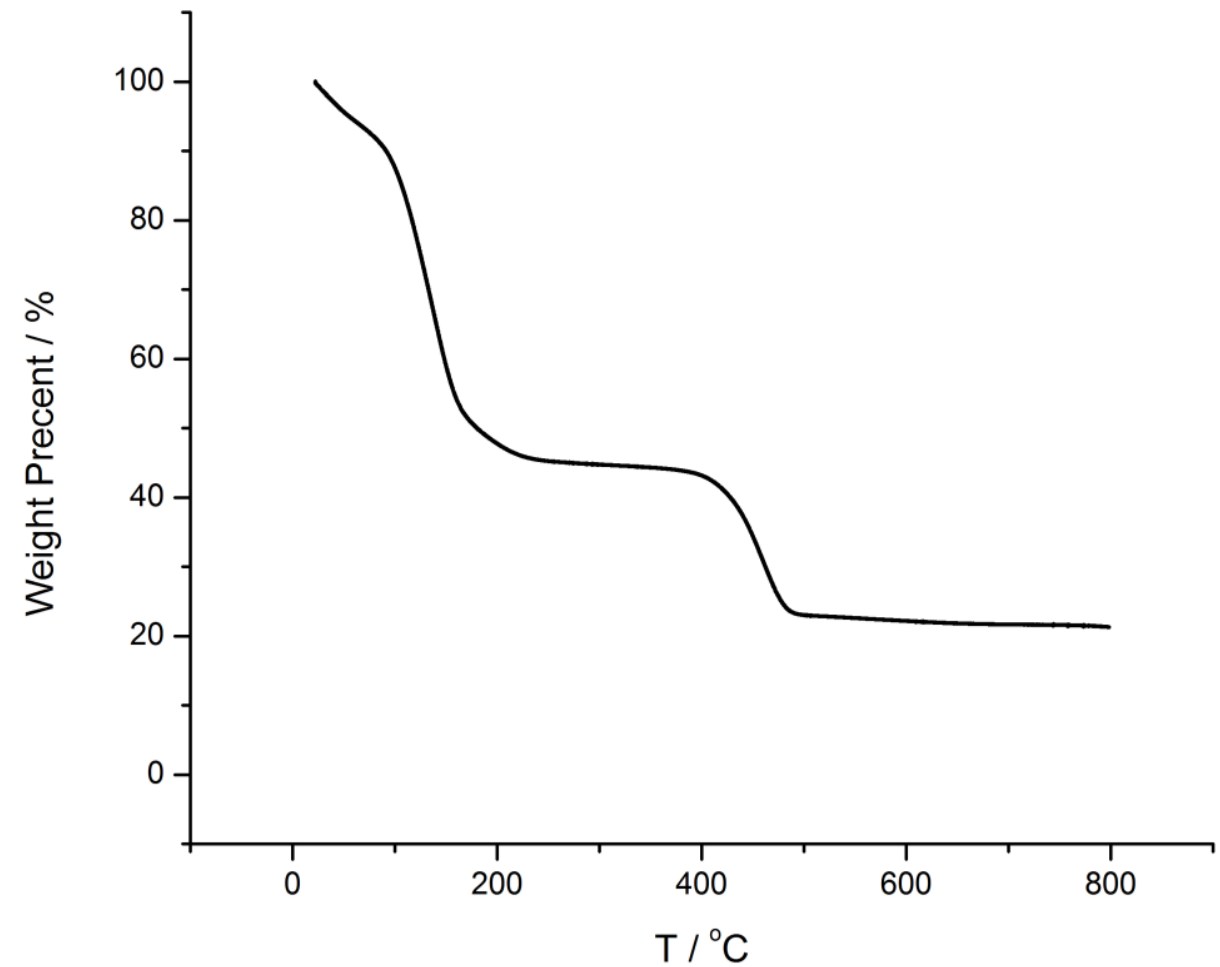

Figure S4. TGA curve of CMOF-5.

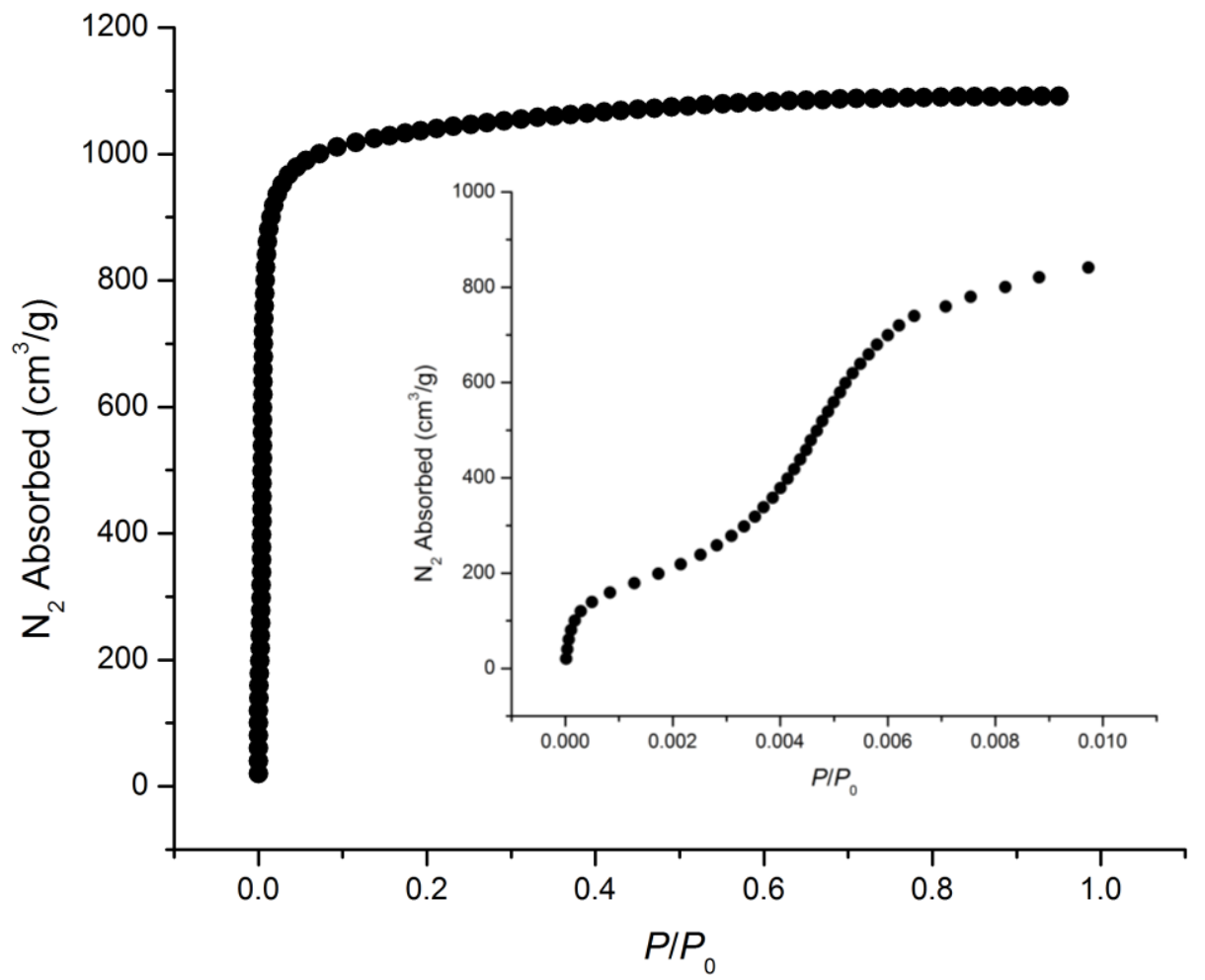

Figure S5. Nitrogen adsorption isotherm for CMOF-5 at $77 \mathrm{~K}$. 

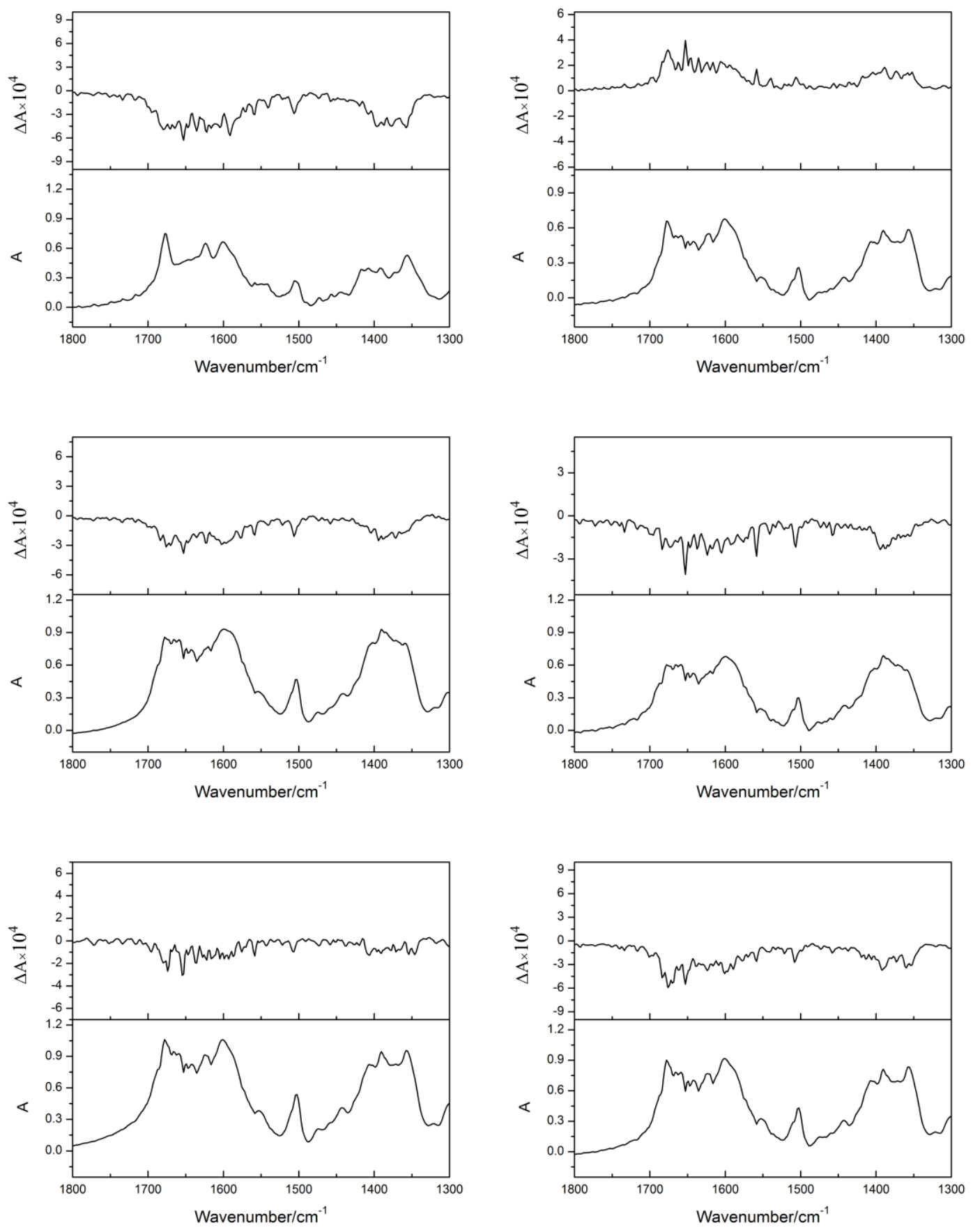

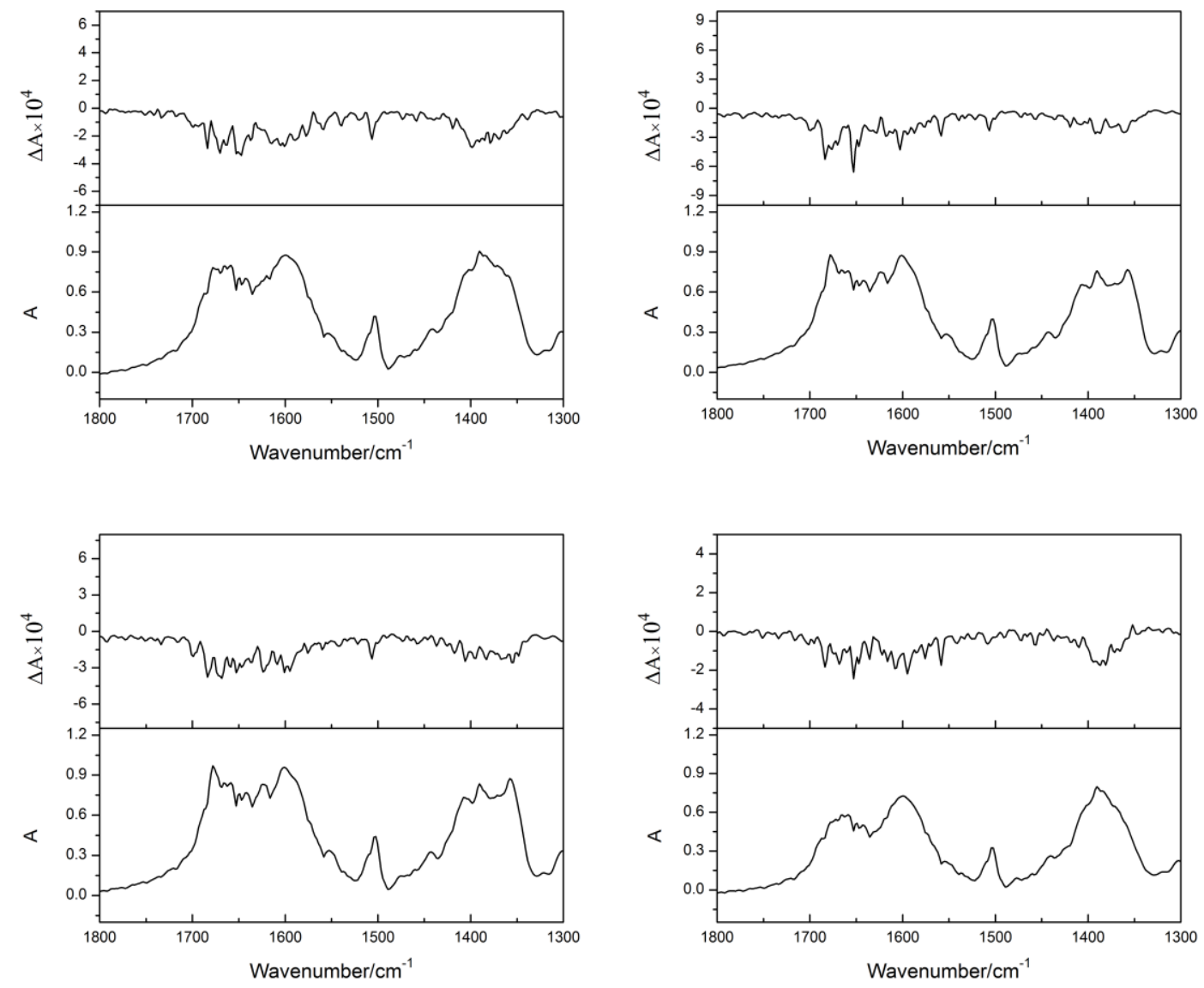

Figure S6. Solid state VCD spectra of $\Lambda$-CMOF-5 (10 crystals measured with $e e=80 \%)$. 

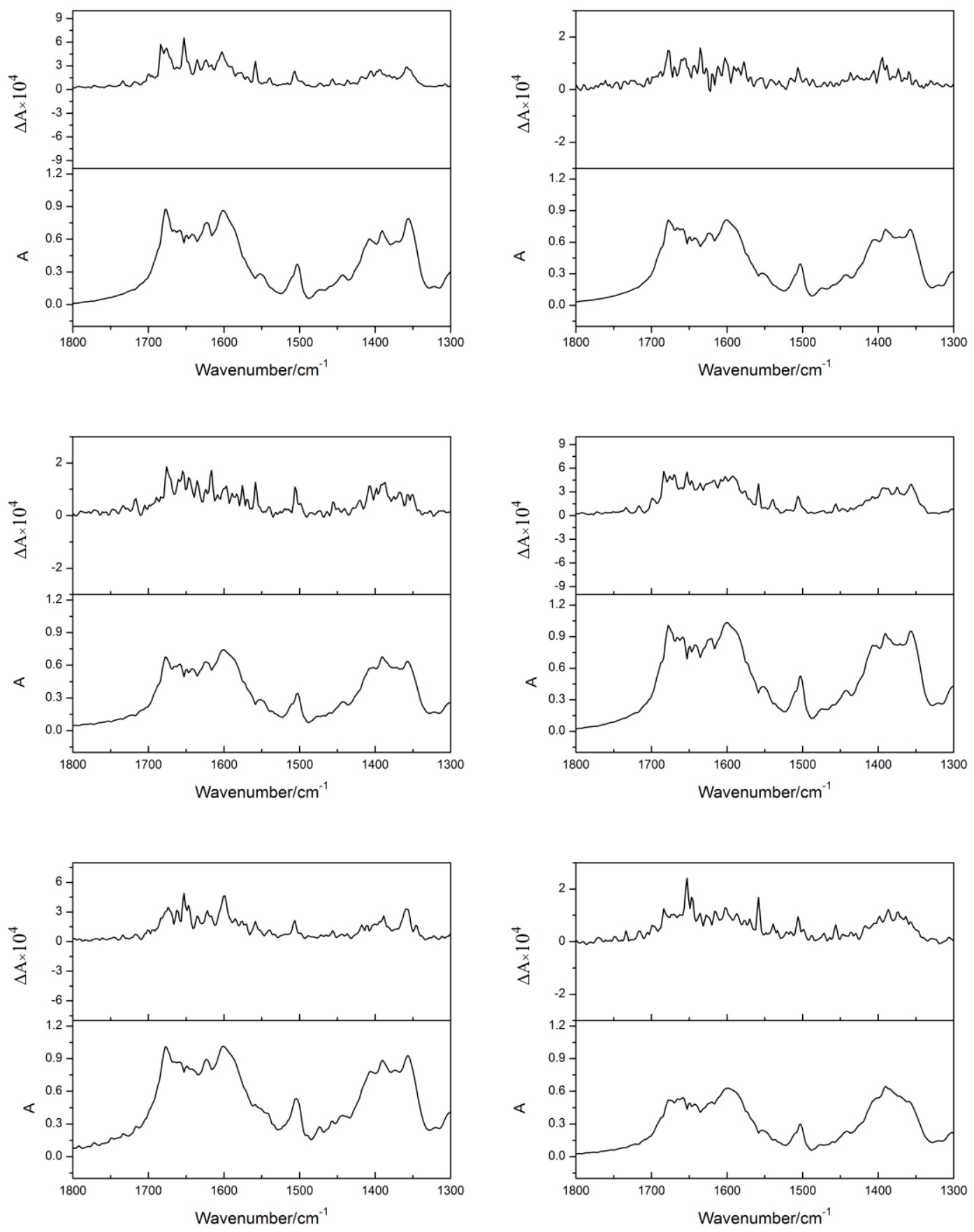

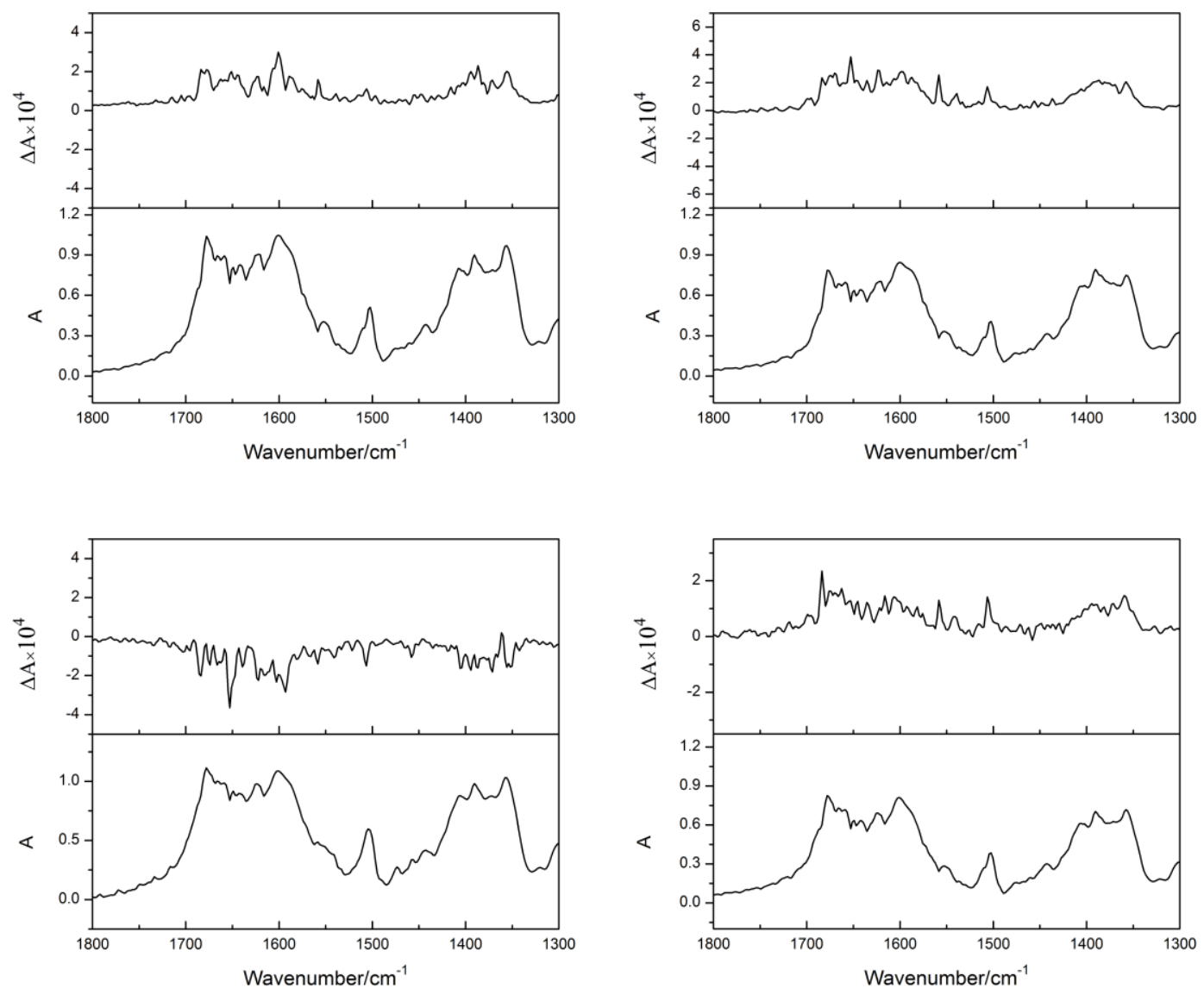

Figure S7. Solid state VCD spectra of $\Delta$-CMOF-5 (10 crystals measured with $e e=80 \%)$. 


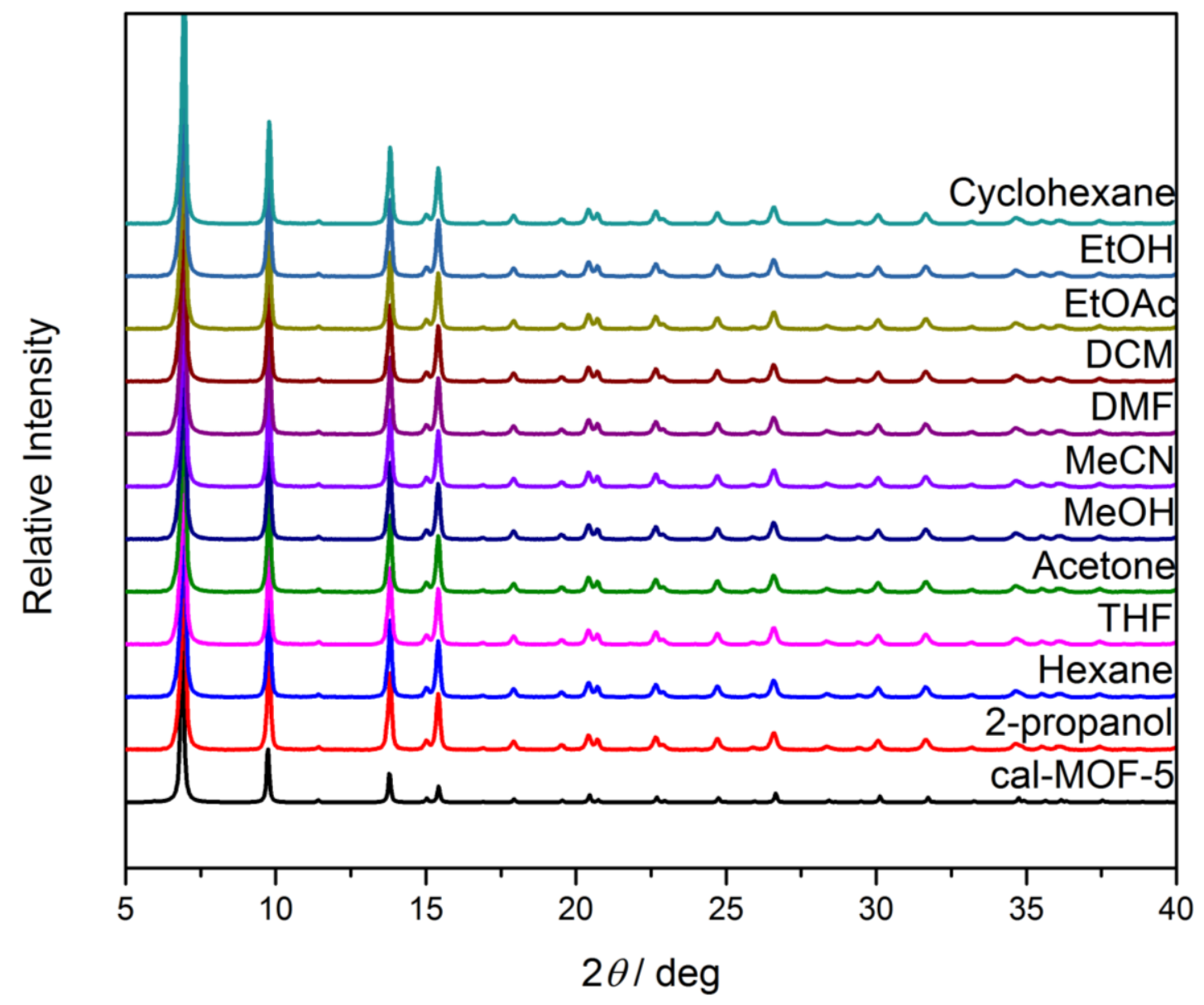

Figure S8. Powder X-ray diffraction (PXRD) patterns for calculated MOF-5 (cal-MOF-5) and CMOF-5 immersed in different solvents. 


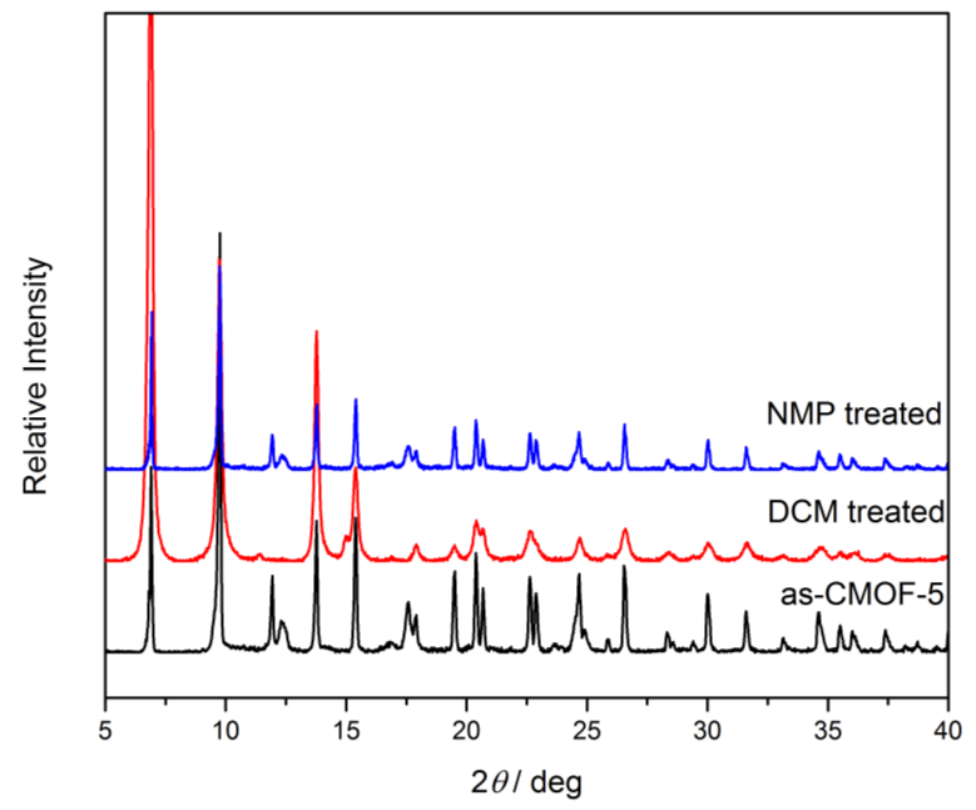

Figure S9. Comparison of powder X-ray diffraction (PXRD) patterns for phase transformation from CMOF-5 to MOF-5 to CMOF-5. 

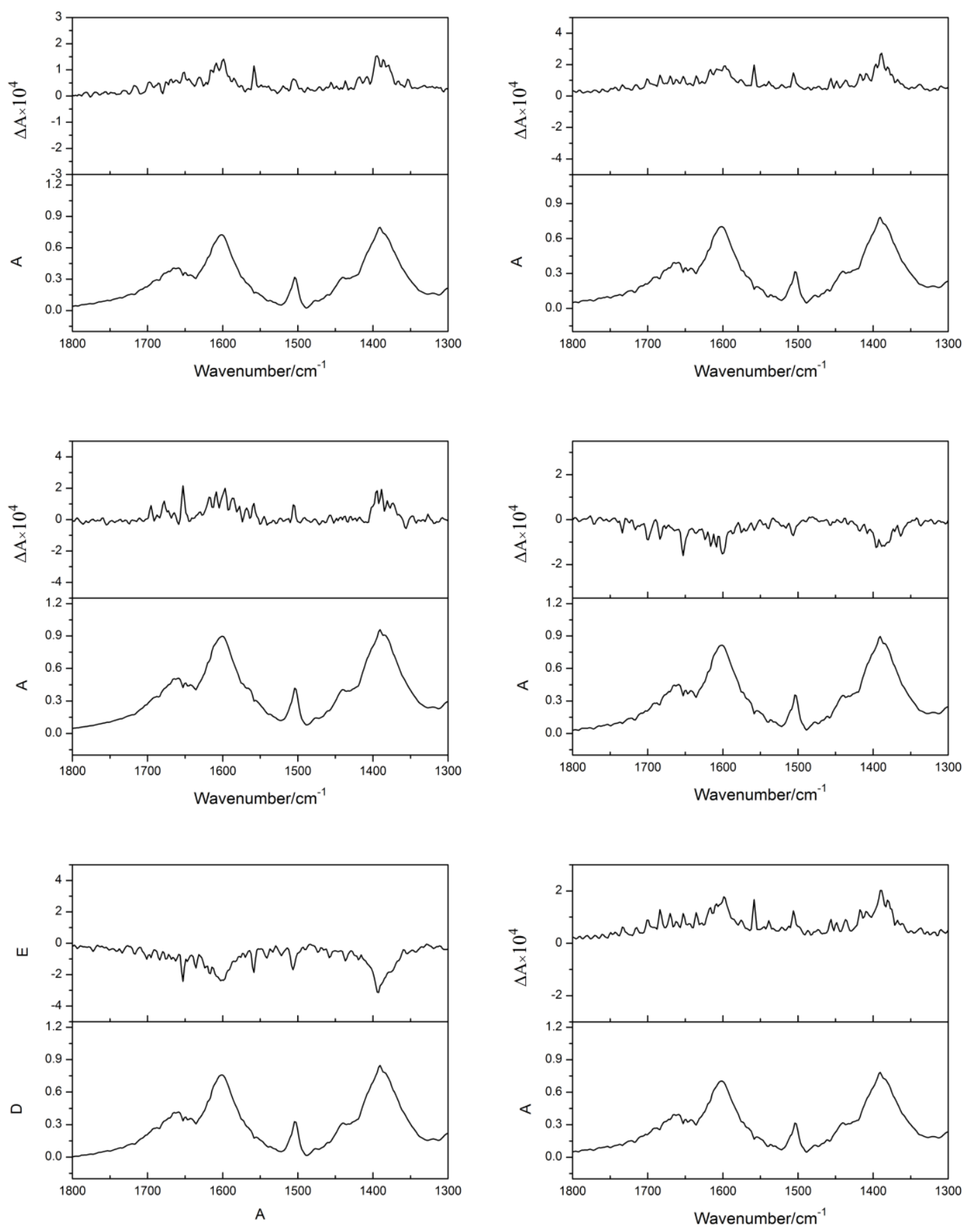

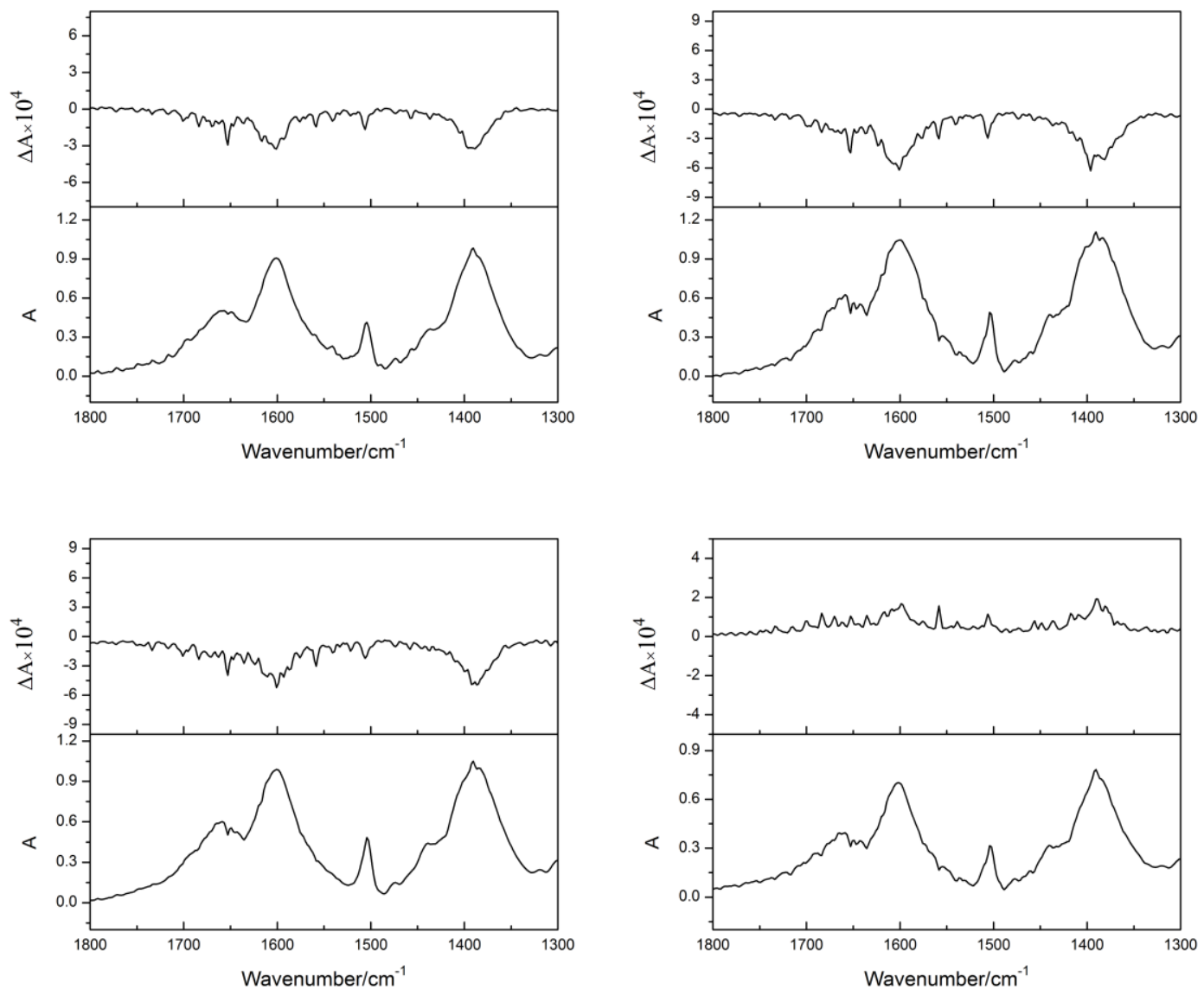

Figure S10. Solid state VCD spectra of MOF-5 after NMP treatment (10 crystals measured with $e e=0$ ). 
Table S1 Crystal data and structure refinement details of the frameworks of CMOF-5 and

\begin{tabular}{cccc}
\multicolumn{4}{c}{$[\mathrm{Zn}(\mathrm{BDC})(\mathrm{NMP})]}$. \\
\hline formula & $\Lambda$-CMOF-5 & $\Delta$-CMOF-5 & {$[\mathrm{Zn}(\mathrm{BDC})(\mathrm{NMP})]$} \\
$\mathrm{fw}$ & $\mathrm{C}_{71.5} \mathrm{H}_{97.5} \mathrm{~N}_{9.5} \mathrm{Zn}_{4} \mathrm{O}_{2}$ & $\mathrm{C}_{71.5} \mathrm{H}_{97.5} \mathrm{~N}_{9.5} \mathrm{Zn}_{4} \mathrm{O}_{2}$ & $\mathrm{C}_{13} \mathrm{H}_{13} \mathrm{NO}_{5} \mathrm{Zn}$ \\
temp $(\mathrm{K})$ & 1711.73 & 1711.73 & 328.61 \\
cryst system & $100(2)$ & $100(2)$ & $228(2)$ \\
space group & $\mathrm{Cubic}$ & $\mathrm{Cubic}$ & Monoclinic \\
$a(\AA)$ & $P 2{ }_{1} 3$ & $P 2{ }_{1} 3$ & $P 2{ }_{1} / c$ \\
$b(\AA)$ & $25.3688(7)$ & $25.3504(6)$ & $7.45310(10)$ \\
$c(\AA)$ & $25.3688(7)$ & $25.3504(6)$ & $13.9080(2)$ \\
$\beta\left({ }^{\circ}\right)$ & $25.3688(7)$ & $25.3504(6)$ & $12.7717(2)$ \\
$V\left(\AA^{3}\right)$ & 90.00 & 90.00 & $103.2200(10)$ \\
$Z$ & $16326.7(8)$ & $16291.2(12)$ & $1288.80(3)$ \\
$\mathrm{Dc}\left(\mathrm{g} \cdot \mathrm{cm}^{-3}\right)$ & 8 & 8 & 4 \\
$\mu\left(\mathrm{mm}{ }^{-1}\right)$ & 0.767 & 0.628 & 1.694 \\
$F(000)$ & 1.694 & 1.536 & 2.843 \\
$\mathrm{R}_{\text {int }}$ & 3732.0 & 3040.0 & 672.0 \\
$\mathrm{GOF}$ & 0.0908 & $-{ }^{-1}$ & 0.0466 \\
$R_{1}(\mathrm{I}>2 \sigma(\mathrm{I}))$ & 1.083 & 1.052 & 1.095 \\
$w R_{2}(\mathrm{all} \mathrm{data)}$ & 0.0956 & 0.0729 & 0.0281 \\
$\triangle \rho_{\text {max }}\left(\mathrm{e} \AA^{-3}\right)$ & 0.2728 & 0.2180 & 0.0727 \\
$\triangle \rho_{\text {min }}\left(\mathrm{e} \AA^{-3}\right)$ & 0.95 & 0.84 & 0.33 \\
\hline
\end{tabular}

${ }^{a}$ HKL file has been generated from detwinned FCF. 